\title{
Limits on the time variation of the electromagnetic fine-structure constant in the low energy limit from absorption lines in the spectra of distant quasars
}

\author{
R. Srianand ${ }^{1}$, H. Chand ${ }^{1}$, P. Petitjean ${ }^{2,3}$ and B. Aracil ${ }^{2}$ \\ ${ }^{1}$ IUCAA, Post Bag 4, Ganeshkhind, Pune - 411007 \\ ${ }^{2}$ Institut d'Astrophysique de Paris - CNRS, 98bis Boulevard Arago, F-75014 Paris, France \\ ${ }^{3}$ LERMA, Observatoire de Paris, 61 avenue de l'Observatoire, F-75014 Paris, Franc屯
}

(Dated: September 19, 2018)

\begin{abstract}
Most of the successful physical theories rely on the constancy of few fundamental quantities (such as the speed of light, $c$, the fine-structure constant, $\alpha$, the proton to electron mass ratio, $\mu$, etc), and constraining the possible time variations of these fundamental quantities is an important step toward a complete physical theory. Time variation of $\alpha$ can be accurately probed using absorption lines seen in the spectra of distant quasars. Here, we present the results of a detailed many-multiplet analysis performed on a new sample of $\mathrm{Mg}$ II systems observed in high quality quasar spectra obtained using the Very Large Telescope. The weighted mean value of the variation in $\alpha$ derived from our analysis over the redshift range $\mathbf{0 . 4} \leq \mathbf{z} \leq \mathbf{2 . 3}$ is $\mathbf{\Delta} \alpha / \alpha=(-\mathbf{0 . 0 6} \pm \mathbf{0 . 0 6}) \times \mathbf{1 0}^{-\mathbf{5}}$. The median redshift of our sample $(\mathrm{z} \simeq 1.55)$ corresponds to a look-back time of 9.7 Gyr in the most favored cosmological model today. This gives a $3 \sigma$ limit, $\mathbf{- 2 . 5} \times \mathbf{1 0}^{-\mathbf{1 6}} \mathrm{yr}^{-\mathbf{1}} \leq(\boldsymbol{\Delta} \alpha / \alpha \boldsymbol{\Delta} \mathbf{t}) \leq+\mathbf{1 . 2} \times \mathbf{1 0}^{-\mathbf{1 6}} \mathrm{yr}^{-\mathbf{1}}$, for the time variation of $\alpha$, that forms the strongest constraint obtained based on high redshift quasar absorption line systems.
\end{abstract}

PACS numbers: 98.80.Es,06.20.Jr,98.62.Ra

Contemporary theories of fundamental interactions allow the fundamental constants to vary as a function of space and time [1]. Therefore, direct measurements of possible time variations of various fundamental quantities are of utmost importance for a complete understanding of fundamental physics. In this letter we investigate the time variation of the fine-structure constant $\left(\alpha=e^{2} / \hbar c=1 / 137.03599976(50)\right.$ 2], where $e$ is the charge of the electron and $\hbar$ the reduced Planck constant measured in the laboratories on Earth). The time evolution of $\alpha$ can be probed in the framework of standard BigBang models using measurements performed at different redshifts $(z)$. The Oklo phenomenon [3] , a natural fission reactor that operated 2 Gyrs ago, or at $z \sim 0.16$, gives the strongest constraint on the variation of $\alpha$ over cosmological time-scales, $[\Delta \alpha / \alpha \Delta t]=(-0.2 \pm 0.8) \times 10^{-17} \mathrm{yr}^{-1}$. At higher redshifts, a possible time dependence will be registered in the form of small shifts in the absorption line spectra seen toward distant quasars 4 as the energy of the atomic transitions depend on $\alpha$. Initial attempts to measure the variation of $\alpha$ were based on the absorption lines of alkali-doublets 5 (AD method). The best constraint obtained using this method is $\Delta \alpha / \alpha=$ $(-0.5 \pm 1.3) \times 10^{-5}[\underline{6}]$. Other methods such as the one using $\mathrm{O}$ III emission lines [7], though more robust, are not sensitive enough to detect small variations in $\Delta \alpha / \alpha$. Investigations based on molecular lines 8 detected in two systems give $\Delta \alpha / \alpha=(-0.10 \pm 0.22) \times 10^{-5}$ and $(-0.08 \pm 0.27) \times 10^{-5}$ at $z_{\text {abs }}=0.2467$ and 0.6847 respectively. Such studies at high-z are elusive due to lack of molecular systems. The generalization of alkali-doublet method, called many-multiplet (MM) method, gives an order of magnitude improvement in the measurement of $\Delta \alpha / \alpha$ compared to AD method $[9]$ by using not only dou- blets but several multiplets from different species. The sensitivity of different line transitions from different multiplets to variations in $\alpha$ were computed using manybody calculations taking into account dominant relativistic effects 10].

In simple terms, MM method exploits the fact that the energy of different line transitions vary differently for a given change in $\alpha$. For example rest wavelengths of $\mathrm{Mg}$ II $\lambda \lambda 2797,2803$ and $\mathrm{Mg}$ I $\lambda 2852$ transitions are fairly insensitive to small changes in $\alpha$ thereby providing good anchor for measuring the systemic redshift. Whereas the rest wavelengths of Fe II multiplets are quite sensitive to small variations in $\alpha$. Thus measuring consistent relative shifts between an anchor and different Fe II lines can in principle lead to an accurate measurement of $\Delta \alpha / \alpha$. The accuracy at which the variation can be measured depends very much on how well absorption profiles can be modeled. For this usually Voigt profiles that are convolved with the instrumental profile and characterized by column density $(N)$, velocity dispersion $(b)$ and redshift in addition to the rest-wavelength of the species are used. In a real spectrum small relative shifts can be introduced by various systematic effects such as inhomogeneities in the absorbing region, poor wavelength calibration, isotopic abundances, and atmospheric dispersion effects, etc 13]. However most of the random systematic effects can be canceled by using a large number of measurements. MM method applied to large samples of quasar absorption lines resulted in the claim for smaller value of $\alpha$ in the past [9], $\Delta \alpha / \alpha=(-0.574 \pm 0.102) \times 10^{-5}$ for $0.2 \leq z \leq 3.7$. The main motivation of the present work is to perform similar analysis using a completely different line fitting code and an independent, uniform, better quality, and well defined data sample. 
The data used in this study were obtained with the Ultra-violet and Visible Echelle Spectrograph (UVES) mounted on the ESO Kueyen $8.2 \mathrm{~m}$ telescope at the Paranal observatory for the ESO-VLT Large Programme "QSO absorption lines". This programme has been devised to gather a homogeneous sample of echelle spectra of 18 quasars, with the uniform spectral coverage, resolution and signal-to-noise ratio $(\mathrm{S} / \mathrm{N})$ suitable for studying the intergalactic medium in the redshift range 1.7-4.5. Spectra of quasars were obtained in service mode observations spread over 4 periods (2 years) covering 30 nights under good seeing conditions $(\leq 0.8$ arcsec) using a slit 1 arcsec wide. The data were reduced using the UVES pipeline, a standard set of procedures implemented in a dedicated context of MIDAS, the ESO data reduction package. However independent consistency checks were made to ensure good sky-background subtraction (better than $1 \%$ accuracy), accurate wavelength calibration (rms in $\Delta \lambda / \lambda$ better than $\sim 4 \times 10^{-7}$ over the full wavelength range of interest, $3100-5400$ and $5450-9000 \AA$ ) and optimum signal to noize $(\mathrm{S} / \mathrm{N} \sim 50-80$ per pixel). We fit the continuum using lower order polynomial that gives $\chi^{2} \simeq 1$. Air-vacuum conversions and heliocentric corrections were done using standard conversion equations 11. The slit was always oriented along the parallactic angle and calibration exposures were taken before or after the scientific exposures. The full descriptions of the data reduction and calibration procedures, sample selection and all the steps involved in the analysis are presented in Chand et al.,(2004) 12].

As a first step we have performed a detailed analysis on simulated data to have a clearer understanding of various possible systematics that can affect the analysis of real data. The results of this exercise are used to validate our procedure and define selection criteria that will minimise systematics in our analysis. Absorption spectra of Mg II and Fe II were simulated for given $N$, and $b$. The spectral resolution and $\mathrm{S} / \mathrm{N}$ ratio were similar to our UVES data. The standard fitting function and coefficients 10 are used to incorporate the effects of $\alpha$ variation. We considered two cases: a simple single component system and a highly blended two-component system. In the highly blended case we restricted the separation between the two components to be always smaller than the velocity dispersion of one of the components. We then fitted the absorption lines in order to recover $\Delta \alpha / \alpha$ introduced in the input spectrum. This exercise is to determine the precision that can be reached using our method and fitting procedure. For this we used the Voigt profile fitting method and standard $\chi^{2}$ statistics to fit the absorption profiles consistently and to determine the best fit value for $\Delta \alpha / \alpha$. The relationships between the input and recovered values of $\Delta \alpha / \alpha$ are shown in Fig. 1] The method works very well in the case of simple single component systems where one expects minimum uncertainties due to systematics. The deviation of the recovered value with
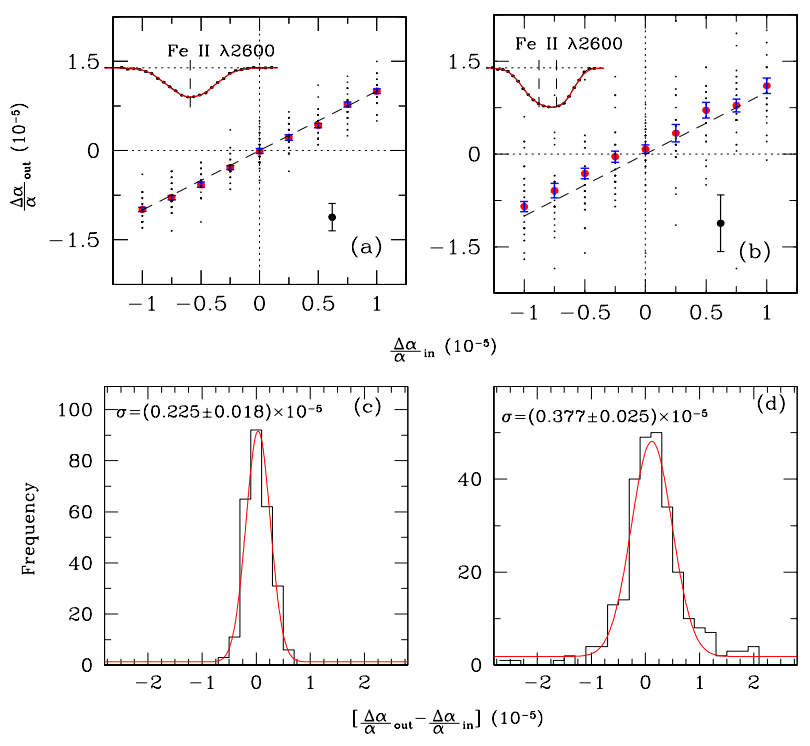

FIG. 1: Absorption spectra of $\mathrm{Mg}$ II and Fe II were simulated for a given set of $N, b$, and spectral resolution similar to our data, introducing spectral shifts corresponding to a given value of $\Delta \alpha / \alpha$. Top panels show the relationship between the input and derived value of $\Delta \alpha / \alpha$ in the case of a single clean component (left-hand side) and a blend of two components (right-hand side). Each realization is performed using random values of $N, b$ and noise keeping the signal-tonoise ratio, wavelength sampling and resolution as in a typical UVES spectrum. Dots are the values from individual realizations and the points with the error bars are the weighted mean obtained from 30 realizations. A typical absorption profile is also shown in these panels. The lower panels give the distribution of the recovered $\Delta \alpha / \alpha$ around the true one. Single (left) and blended (right) cases are considered respectively. Best fitted Gaussian distributions are over-plotted.

respect to the true one distributes like a Gaussian with $\sigma=0.23 \times 10^{-5}$. This shows that $\Delta \alpha / \alpha$ can be constrained with an accuracy of $0.07 \times 10^{-5}$ even when we use 10 such systems. As expected the scatter is more in the case of blended systems $\left(\sigma=0.38 \times 10^{-5}\right)$. Similar analysis on two components with separations larger than individual $b$ values gives $\sigma=0.261 \times 10^{-5}$. Indeed, in this case, the separation of the components is large enough so that each component is clearly recognized by the fitting procedure. In such a situation the corresponding errors are very much the same as for individual components. The conclusion is that it is better to select single component or not heavily blended systems, that is the components are separated by more than their $b$ values, to obtain the most reliable results. We notice from our simulations that systems with weak lines have large uncertainties in the measured $\Delta \alpha / \alpha$ and should be avoided from the analysis. Finally, the data used in this analysis have a median $\mathrm{S} / \mathrm{N}$ ratio of 70 per pixel, a factor of two better than that used in earlier studies. Based on 


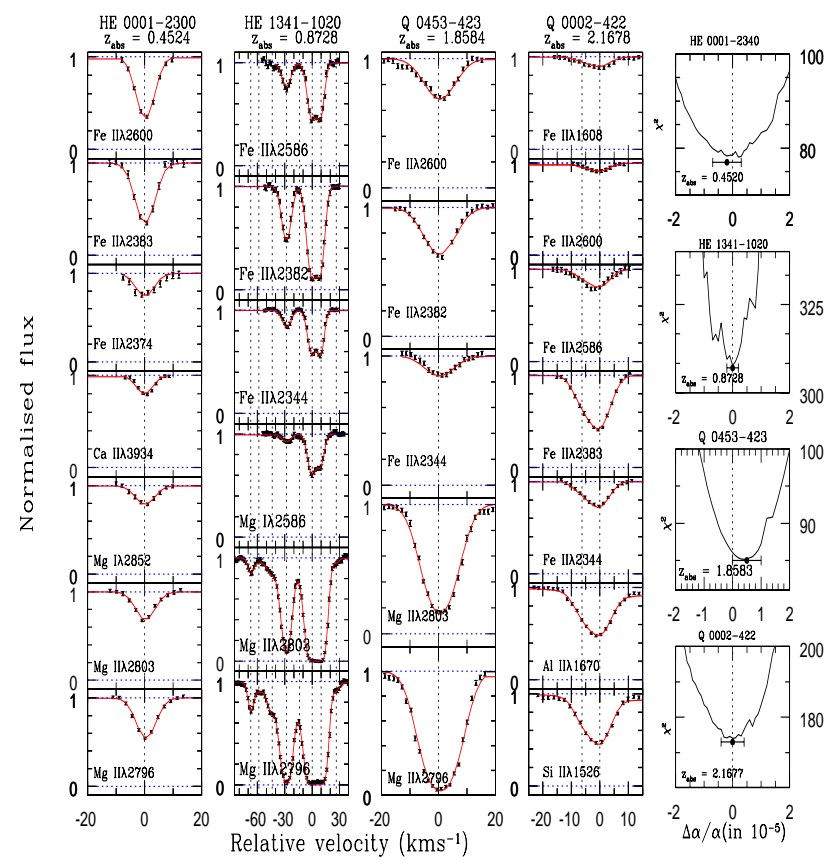

FIG. 2: Voigt profile fits to 4 randomly chosen systems (out of 23) in our sample are shown in the first 4 columns from the left. The quasar name and absorption redshifts are given on top of the panels. The points with the error bars are the observed data and the continuous curve is the fit obtained using multicomponent Voigt profile decomposition. The locations of different components are marked with vertical dotted lines. The plots in the right most column demonstrate how $\Delta \alpha / \alpha$ is extracted from these systems. We plot $\chi^{2}$ as the function of $\Delta \alpha / \alpha$. The minimum in this curve (marked with a dot) gives the best-fit value of $\Delta \alpha / \alpha$ and the error in this measurement (error-bar around the dot) is based on the standard statistical method of computing errors from $\Delta \chi^{2}=1$.

the simulations we expect this enhancement in the $\mathrm{S} / \mathrm{N}$ ratio to provide a roughly a factor two improvement in the $\Delta \alpha / \alpha$ measurements.

In summary, based on the results from the simulations, we apply the following selection criteria to derive reliable $\Delta \alpha / \alpha$ : (i) we consider only species with similar ionization potentials ( $\mathrm{Mg}$ II, Fe II, Si II and $\mathrm{Al}$ II) as they are most likely to originate from similar regions in the cloud; (ii) we avoid absorption lines that are contaminated by atmospheric lines; (iii) we consider only systems that have $N$ (Fe II $) \geq 2 \times 10^{12} \mathrm{~cm}^{-2}$ which ensures that all the standard Fe II multiplets are detected at more than $5 \sigma$ level; (iv) we demand that at least one of the anchor lines is not saturated so that the redshift measurement is robust; (v) we also avoided sub-DLAs (i.e $N(\mathrm{H} \mathrm{I}) \geq 10^{19}$ $\mathrm{cm}^{-2}$ ) as these systems may have ionization and chemical inhomogeneities; (vi) we do not consider strongly saturated systems with large velocity spread (complex blends); however in such systems whenever we find a well detached satellite components we include these compo- nents in the analysis; (vii) finally, based on the component structure resulting from the Voigt profile fits of systems that are not complex blends, we retain only systems for which the majority of components are separated from its neighboring components by more than the $b$ parameters. Application of the above conditions resulted in 23 systems for performing $\Delta \alpha / \alpha$ measurements $(6$ single component systems, 6 well separated doubles, 6 systems with 3 components with at least one well detached from the rest, and 5 systems with more than 3 components) .

In our analysis we use the fact that relative shifts between lines from the same species (say Fe II multiplets) are insensitive to $\Delta \alpha / \alpha$. We first fit all the Fe II lines (except for Fe II $\lambda 1608$ ) simultaneously using laboratory wavelengths. This allows us to find out about (i) bad pixels, (ii) unknown contaminations and (iii) the velocity component structure in Fe II. Defective absorption lines detected are removed from the $\Delta \alpha / \alpha$ analysis. Similar exercise was carried out for $\mathrm{Mg}$ II doublets and other anchors. Based on these preliminary fits a first set of parameters is generated to start the Voigt profile fitting procedure that includes $\Delta \alpha / \alpha$ variations. We illustrate the method in Fig. 2 using 4 randomly chosen systems from our sample. We vary $\Delta \alpha / \alpha$ ranging from $-5.0 \times 10^{-5}$ to $5.0 \times 10^{-5}$ in step of $0.1 \times 10^{-5}$, and each time fit all the lines together. $\chi^{2}$ minima obtained for each of these fits are plotted as a function of $\Delta \alpha / \alpha$ (right most panel in Fig. (2). The value of $\Delta \alpha / \alpha$ at which this $\chi^{2}$ is minimum is accepted as the measure of best possible $\Delta \alpha / \alpha$ value. Following standard statistical procedure we assign $1 \sigma$ error bar to the best fitted value of $\Delta \alpha / \alpha$ by computing the change in $\Delta \alpha / \alpha$ implying $\Delta \chi^{2}=\chi^{2}-\chi_{\min }^{2}=1$. We always consider two different models: (i) one in which $b$ parameters for all the species are the same and (ii) one in which $b$ parameters for different species are different. In all systems we notice that the derived $\Delta \alpha / \alpha$ using both cases are consistent with one another within $1 \sigma$ uncertainty. We use the result that has a lower reduced $\chi^{2}$ for our final analysis.

Results obtained for the 23 systems in our sample and that from the literature are summarized in Fig. 3 The shaded region passing through most of the error bars is the weighted mean (with $1 /$ error $^{2}$ weights) and its $3 \sigma$ error from our sample. It is clear that most of our measurements are consistent with zero within the uncertainties. The simple mean, weighted mean and standard deviation around the mean obtained for our sample are $(-0.02 \pm 0.10) \times 10^{-5},(-0.06 \pm 0.06) \times 10^{-5}$ and $0.41 \times 10^{-5}$ respectively. The corresponding values are $(0.01 \pm 0.15) \times 10^{-5},(-0.08 \pm 0.07) \times 10^{-5}$ and $0.26 \times 10^{-5}$ respectively when we restrict our analysis to the single and well detached two component systems (12 in total). These systems have maximum influence in the weighted mean of the whole sample. Interestingly, error on the weighted mean and standard deviation around the mean from these systems are very close to the predictions from 


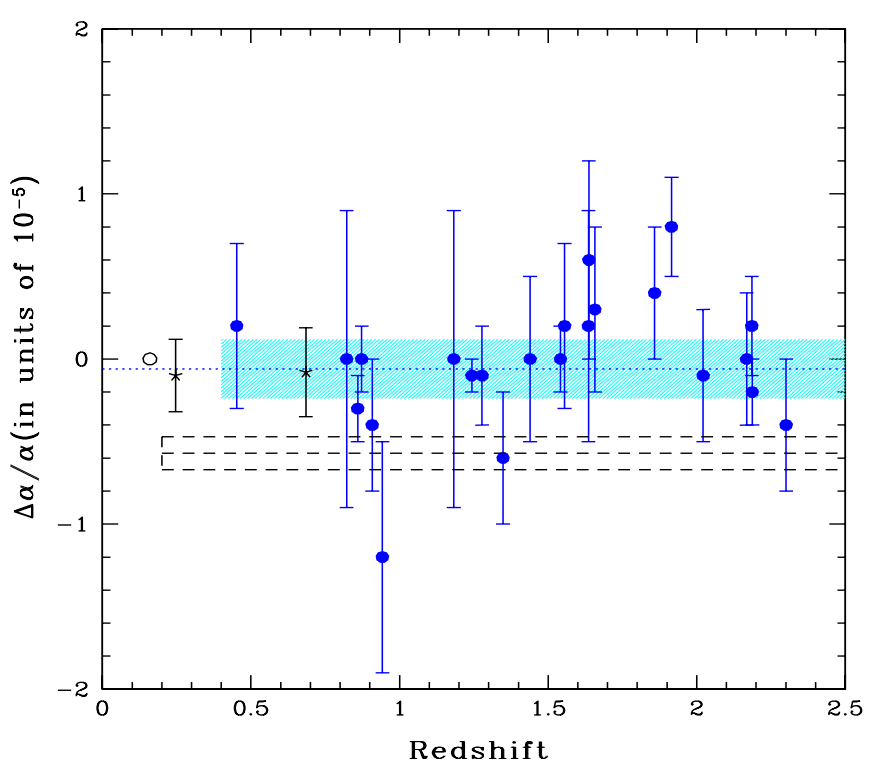

FIG. 3: The measured values of $\Delta \alpha / \alpha$ from our sample (filled circles) are plotted against the absorption redshifts of $\mathrm{Mg}$ II systems. Each point is the best fitted value obtained for individual systems using $\chi^{2}$ minimization as demonstrated in Fig. 2] The open circle and stars are the measurement from Oklo phenomenon [3] and from molecular lines [8] respectively. The weighted mean and $1 \sigma$ range measured by Murphy et al.(2003) are shown with the horizontal long dashed lines. Clearly most of our measurements are inconsistent with this range. The shadow region marks the weighted mean and its $3 \sigma$ error obtained from our study.

our simulations. In all cases weighted mean values are consistent with all the data points with a reduced $\chi^{2} \simeq 1$.

Thus our study gives a more stringent $3 \sigma$ constraint of $-0.24 \leq \Delta \alpha / \alpha\left(\right.$ in $\left.10^{-5}\right) \leq+0.12$ over the redshift range of $0.4 \leq z \leq 2.3$. The median redshift of the whole sample is 1.55 which corresponds to a look-back time of 9.7 Gyr in the most favored cosmological model today $\left(\Omega(\right.$ total $)=1, \Omega_{\Lambda}=0.7, \Omega($ matter $)=0.3$, and $\mathrm{H}_{0}$ $\left.=68 \mathrm{~km} \mathrm{~s}^{-1} \mathrm{Mpc}^{-1}\right)$. This gives a $3 \sigma$ constraint on the time variation of $\Delta \alpha / \alpha$ to be $-2.5 \times 10^{-16} \mathrm{yr}^{-1} \leq$ $(\Delta \alpha / \alpha \Delta t) \leq 1.2 \times 10^{-16} \mathrm{yr}^{-1}$. As can be seen from Fig. 3 our results are consistent with the constraints from Oklo phenomenon and from the molecular lines. However, our study does not support the claims by previous authors of a statistically significant change in $\Delta \alpha / \alpha$ with cosmic time at $z>0.5$ using MM method. In any case our measurement still does allow smaller variations in excess of what is found based on the Oklo phenomenon. Future very high resolution $(\mathrm{R} \sim 100,000)$ spectroscopic studies are needed to probe the variations in $\alpha$ with much better accuracy.

This work is based on observations collected during programme 166.A-0106 (PI: Jacqueline Bergeron) of the European Southern Observatory with the Ultra-violet and Visible Echelle Spectrograph mounted on the $8.2 \mathrm{~m}$ Kueyen telescope operated at the Paranal Observatory, Chile. HC thanks CSIR, INDIA for the grant award No. 9/545(18)/2KI/EMR-I. RS thanks CNRS/IAP for the hospitality. We gratefully acknowledge support from the Indo-French Centre for the Promotion of Advanced Research (Centre Franco-Indien pour la Promotion de la Recherche Avancée) under contract No. 3004-A.

* Electronic address: anand@iucaa.ernet.in, hcverma@iucaa.ernet.in, p

[1] Uzan, J. P. Rev. Mod. Phys. 75, 403-455 (2003)

[2] Mohr, P. J., Taylor, B. N. Rev. Mod. Phys. 72, 351-495 (2000)

[3] Fujii, Y., et al., Nuc. Phy. B 573, 377-401 (2000)

[4] Savedoff, M. P., Nature 178, 688-689(1956); Bahcall, J.N., Sargent, W. L. W., Schmidt, M., Astrophys. J. 149, L11-L15 (1967)

[5] Wolfe, A. M., Brown, R. L., Roberts, M. S., Phys. Rev. Lett. 37, 179-181 (1976); Cowie, L. L., Songaila, A. Astrophys. J. 453, 596-598 (1995); Varshalovich, D. A., Panchuk, V. E., Ivanchik, A. V., Astron. Lett 22, 6-13 (1996)

[6] Murphy, M. T., Webb, J., Flambaum, V., Prochaska, J. X., Wolfe, A. M., Mon. Not. R. Soc. 327, 1237-1243 (2001)

[7] Bahcall, J. N., Steinhardt, C. L., Schlegel, D., Astro;hys. J., 600, 520-543 (2004)

[8] Murphy, M. T. et al., Mon. Not. R. Astron. Soc. 327, 1244 (2001)

[9] Dzuba,V.A., Flambaum, V. V., Webb, J. K., Phys. Rev. Lett. 82, 888-891 (1999); Webb, J. K., et al. Phys. Rev. Lett. 87, 091301 (2001); Murphy, M. T., Webb, J. K., Flambaum, V. V., Month. Not. R. Astron. Soc. 345, 609638 (2003)

[10] Dzuba,V.A., Flambaum, V. V., Webb, J. K., Phys. Rev. A 59, 230-237 (1999); Dzuba,V.A., Flambaum, V. V., Kozlov M. G., Marchenko, M., Phys. Rev. A 66, 022501 (2002)

[11] Edlén, B., Metrologia, 2,71-80 (1966); Stumpff, P., Astron. Astrophys. Supl. Series, 41,1 (1980)

[12] Chand, H., Srianand, R., Petitjean, P., Aracil, B., astro-ph/0401094 (2004)

[13] Murphy, M. T., Webb, J. K., Flambaum, V. V., Churchill, C. W., Prochaska, J. X. Month. Not. R. Astron. Soc. 327, 1223-1236 (2001) 